\title{
Retrospective Review of Cerebral Mycotic Aneurysms in 26 Patients: Focus on Treatment in Strongly Immunocompromised Patients with a Brief Literature Review
}

\author{
L.M. Allen, A.M. Fowler, C. Walker, C.P. Derdeyn, B.V. Nguyen, A.N. Hasso, B.V. Ghodke, G.J. Zipfel, D.T. Cross III, and C.J. Moran
}

\begin{abstract}
BACKGROUND AND PURPOSE: Cerebral mycotic aneurysms are a rare and deadly type of aneurysm that have no definitive treatment guidelines. Our purpose was to retrospectively review known or suspected cases of CMA in order to identify patient populations that may be associated with higher morbidity and mortality. We hope that the identification of patients with these risk factors will lead to early stratification upon presentation, and more urgent treatment of their CMAs. We also hoped to identify any benefit or complication that was specific to either the endovascular or neurosurgical repair of CMAs.
\end{abstract}

MATERIALS AND METHODS: A retrospective multi-institutional study was performed examining cases of CMA during a 15-year period. Patients were considered strongly immunocompromised if there were long-term severely immunocompromised states: AIDS, chemotherapy, or steroid immunosuppression. Patients were excluded if angiographic findings suggested an alternative diagnosis or if an infectious etiology was unknown. Antibiotics were considered "noninvasive treatment." Endovascular and neurosurgical repair were considered "invasive treatment." Data were recorded by reviewing electronic medical records and imaging reports.

RESULTS: Twenty-six patients with $40 \mathrm{CMAs}$ were included. Three patients were considered strongly immunocompromised and presented with 4 CMAs, which demonstrated larger average size and more rapid growth; 3 of these patients' aneurysms were treated invasively in the acute period, with the one that was not ruptured causing death. Technical success (aneurysm occlusion without rupture or recanalization) and clinical success (no neurologic complication attributable to the intervention) were obtained equally endovascularly and neurosurgically. Clipping was aborted in favor of coiling for 1 patient. Anticoagulation needed reversal before 2 patients underwent craniotomy for clipping after valve replacement. For CMAs treated with antibiotics alone with angiographic follow-up ( $n=11)$, initial aneurysm size was unrelated to persistence and $64 \%$ completely regressed.

CONCLUSIONS: We recommend initial invasive treatment for CMAs in strongly immunocompromised patients. Testing for underlying immunocompromised states is warranted in patients with CMAs. Endovascular treatment is favored over neurosurgical treatment in patients requiring acute cardiac valve repair due to delays with anticoagulation reversal.

ABBREVIATIONS: CMA = cerebral mycotic aneurysms; ECA = external carotid artery; ICP $=$ intracranial pressure; IE $=$ infective endocarditis; $I \mathrm{PH}=$ intraparenchymal hemorrhage

C erebral mycotic aneurysms or infectious intracranial aneurysms represent less than $5 \%$ of all intracerebral aneurysms. ${ }^{1}$ They are most commonly seen in patients with septicemia and HIV/AIDS and are a particularly well-known complication of infective endocarditis. Intravenous drug abuse and "relative immu-

Received February 27, 2012; accepted after revision July 12.

From the Department of Radiological Sciences (L.M.A., B.V.N., A.N.H.), University of California, Irvine, Orange, California; Mallinckrodt Institute of Radiology and Neurointerventional Radiology (A.M.F., C.P.D., G.J.Z., D.T.C., C.J.M.), Washington University, St. Louis, Missouri; and Departments of Radiology and Neurological Surgery, (C.W., B.V.G.), University of Washington Medical Center, Seattle, Washington. Please address correspondence to Laura Allen, MD, UCLA Radiology, 757 Westwood Plaza, Suite 1621, Los Angeles, CA 90095-1721; e-mail: Lmallen0402@hotmail.com http://dx.doi.org/10.3174/ajnr.A3302 nocompromised" states such as diabetes are becoming more commonly associated with CMAs. ${ }^{2-4}$ Studies report $1 \%-10 \%$ of patients with IE have CMAs; and of patients with CMAs, approximately $65 \%$ have IE. ${ }^{3,5}$ In "strongly immunocompromised" patients, CMAs are prone to more rapid growth and rupture. ${ }^{6-8}$ If there is direct meningeal extension of infection, CMAs are often located more proximally than their usual location at distal branch points. In addition, CMAs from atypical infections, especially fungal infections, are particularly lethal. ${ }^{6-8}$

Many believe that endovascular repair should be the treatment of choice for CMAs if the patient is to have valve replacement for IE. ${ }^{5}$ If the patient had recent valve replacement, craniotomy may be contraindicated due to anticoagulation for either mechanical or bioprosthetic valves. If craniotomy is performed before valve 
replacement, there is greater risk for perioperative heart failure compared with endovascular treatment. Endovascular coiling has been successfully performed as early as 1 day before valve replacement. ${ }^{9,10}$ If there are anticoagulation or heart failure concerns, endovascular repair is thought to be a more prudent option than neurosurgical treatment, though, to our knowledge, there are no current prospective data supporting this.

A review of the relevant literature shows no widely accepted guidelines for treating CMAs. Several authors recommend only neurosurgical treatment for ruptured CMAs with intraparenchymal hemorrhage and mass effect. ${ }^{11-13}$ There are no prospective randomized trials comparing endovascular and neurosurgical repair. Endovascular repair is becoming more popular and has been recommended by some to be the treatment of choice. ${ }^{14}$ Prior opinion was that the introduction of foreign material into infected vasculature increases infection and rupture rates. ${ }^{15}$ However, many reports, including a recent literature review that examined 287 cases of CMAs, 46 of which were treated endovascularly, found no postprocedural infections or abscesses for the endovascularly treated patients. ${ }^{5}$ There have only been 3 reported cases of infection after CMA coiling, and there has also been successful coiling in the setting of active bacteremia. ${ }^{16}$

We retrospectively reviewed known or suspected cases of CMA to better improve management, particularly for strongly immunocompromised patients. Technical success for neurosurgical and endovascular repair was analyzed as well as patient risk factors that may lead to increased morbidity and mortality.

\section{MATERIALS AND METHODS}

Multi-institutional review board approval was obtained, and a waiver of informed consent was granted for this Health Insurance Portability and Accountability Act-compliant study. A retrospective nonrandomized study was performed at the University of California, Irvine Medical Center, Department of Radiologic Sciences (2 patients); Mallinckrodt Institute of Radiology, Washington University (13 patients); and the University of Washington Medical Center, Department of Radiology (11 patients). All known or suspected CMA cases were reviewed to include adult patients. Patients were excluded if there was a lack of angiographic findings suggesting CMA or a lack of infectious etiology or if a detailed angiographic study, either catheter angiography or CT angiography, was not performed.

For the purpose of this study, intravenous antibiotics were considered "noninvasive" treatment and endovascular and neurosurgical repair were considered "invasive" treatments. We defined "acute" valve replacement as that occurring within the first 6 weeks after the IV antibiotic initiation. Patients with long-term severe immunosuppression (ie, those with AIDS, those receiving chemotherapy, or those undergoing steroid immunosuppression) were considered strongly immunocompromised. Patients with conditions such as chronic renal failure, diabetes, and chronic alcohol abuse were considered "relatively immunocompromised."

The following parameters were recorded after review: patient age, sex, presenting symptoms, initial imaging findings, comorbidities, presence of IE, cardiac valve-replacement surgery, timing of valve surgery, likely source of infection, status of blood cultures and IV antibiotic administration, number of CMAs, location of CMAs, size of CMAs, record of invasive intervention, technical and clinical success of the intervention, patient morbidity and mortality, and aneurysm size on follow-up imaging. For aneurysm size, the largest dimension was used. In patients whose aneurysm size varied during the course of treatment, the largest measured size was used. Technical and clinical success of the intervention was defined as successful exclusion of the aneurysm without recanalization or repeat bleed, no intraoperative or immediate postoperative rupture, and absence of clinical complications in the immediate postoperative period attributable to the intervention.

\section{RESULTS}

\section{Patient Parameters}

All patients received intravenous antibiotics. Patient 4 had a CTA but was unable to have a catheter angiogram before he died. Limited information was available about initial symptoms in patients 4,15 , and 26. Patient 26 had a remote history of IE with subsequent valve replacement and presented with an incidental CMA years later; data regarding his initial presentation were limited. Follow-up information was not available for patient 15. Information on the sizes of the aneurysm of patient 10 and 1 aneurysm in patient 25 was unavailable. The average patient age was 45 years (range, 26-77 years). Most patients were men (18/26, 69\%). The initial presenting symptom was known in $81 \%$. The most common symptoms were headache $(13 / 21,62 \%)$, focal neurologic deficit $(9 / 21,43 \%)$, and fever $(4 / 21,19 \%)$. Initial imaging findings included subarachnoid hemorrhage (10/25, 40\%), septic infarct $(9 / 25,36 \%)$, and IPH $(8 / 25,32 \%)$.

Sixty percent $(15 / 25)$ of patients had ruptured CMAs, and most had IE (19/26, 73\%). The most common pathogen from blood cultures was Staphylococcus aureus, followed by Streptococcus species. Common risk factors were intravenous drug abuse, poor dental hygiene, or a recent dental procedure. Three patients were considered strongly immunocompromised on the basis of the long duration of a severely immunocompromised state: patient 7 from AIDS, patient 22 from chronic steroid use for Crohn disease, and patient 24 from chemotherapy for advanced multiple myeloma.

\section{Patients Requiring Cardiac Valve Repair}

Ten of the $26(38 \%)$ patients needed cardiac valve replacement from IE, which represented 52\% (10/19) of all patients with IE. Five patients underwent acute valve replacement, and patients 10 and 22 needed anticoagulation reversal, which delayed craniotomy.

\section{Aneurysm Parameters}

Forty CMAs were identified among 26 patients. The most common location was the MCA and its branches (31/40, 78\%). The next most common locations were the anterior cerebral artery and ICA. Aneurysm size was available for 95\% (38/40) and ranged from 1 to $15 \mathrm{~mm}$ (mean, $5 \mathrm{~mm}$; median, $4 \mathrm{~mm}$ ). Aneurysms were multiple in $38 \%(10 / 26)$.

There was an equal incidence of endovascular $(n=9)$ and neurosurgical repair $(n=9)$. Seven aneurysms were treated with 


\section{Aneurysm Response to Antibiotic Treatment}

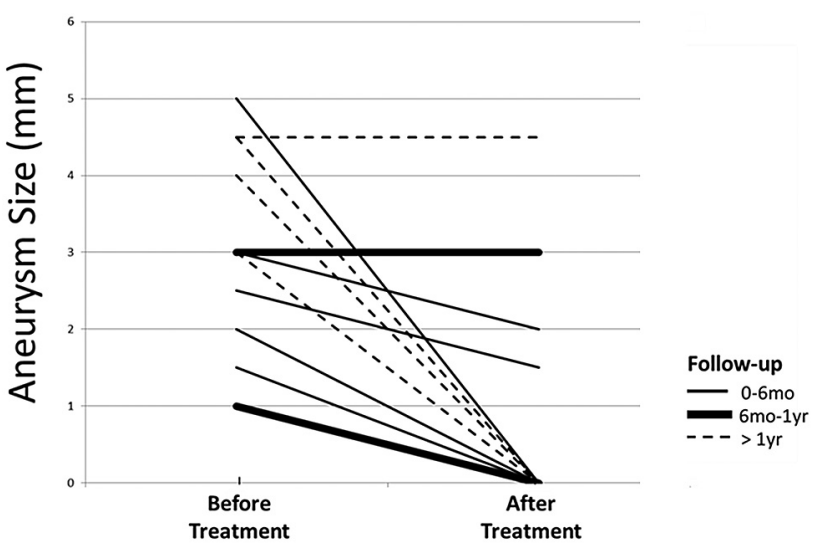

FIG 1. Aneurysm response to antibiotic treatment as depicted in a before-and-after graph. The thin solid line represents aneurysms with a last known angiographic follow-up of $0-6$ months. The thick solid line represents aneurysms with a last known angiographic follow-up of 6 months to 1 year. The dashed line represents aneurysms with a last known angiographic follow-up of $>1$ year. There was no apparent relationship between initial aneurysm size and aneurysm persistence.

coiling ( 1 with a stent scaffold), 2 with gluing, 5 with clipping, 1 with radial artery bypass, 2 with ECA-ICA bypass, and 1 with coagulation.

\section{Antibiotic Treatment Alone}

Of the 22 CMAs treated with antibiotics alone, 50\% had angiographic follow-up. The other half did not have follow-up due to patient death. Follow-up ranged from 1 month to 4 years, and $64 \%(n=7)$ completely resolved, $18 \%(2 / 11)$ decreased in size, and $18 \%(2 / 11)$ were stable. Persistence appeared unrelated to initial aneurysm size as illustrated in a before-and-after graph (Fig 1). CMAs in 4 patients (patients 5, 7, 9, and 22) enlarged during their treatment; patients 7 and 22 were strongly immunocompromised.

\section{Strongly Immunocompromised Patients}

Patients 7, 22, and 24 were strongly immunocompromised and were classified as such due to a long-term severely immunocompromised state. These patients presented with the largest and most rapidly growing CMAs. Average aneurysm size was $11 \mathrm{~mm}$ $(n=4)$.

Patient 7 had AIDS and coccidioidal meningitis. Initially he had a right 8-mm M1 MCA aneurysm, which was successfully coiled with stent scaffolding. Within 7 days between angiograms, he developed a 13-mm anterior communicating artery aneurysm (Fig 2). This was not treated invasively and ruptured, causing his death.

Patient 22, with Crohn disease and chronic steroid immunosuppression, presented with a multilobed left M2 MCA aneurysm, which grew from 6 to $14 \mathrm{~mm}$ within 13 days. He presented with a large septic infarct and was treated initially by clipping, which was aborted after brain herniation out of the craniotomy site secondary to increased ICP. This was successfully coiled (Fig 3).

Patient 24 was on chemotherapy for multiple myeloma and had invasive aspergillosis of the orbit and sinuses and developed an irregular 13-mm left cavernous ICA aneurysm, which was successfully coiled.

Five patients exhibited states of relative immunocompromise, which included chronic renal failure, diabetes, and chronic alcohol abuse. The number of relatively immunocompromised patients in our study is likely greater, given that information on chronic medical conditions in several patients was unobtainable either due to lack of prior medical care or unobtainable patient history.

\section{DISCUSSION}

Our study is concurrent with most reported prior demographics with an average patient age range from 35 to 55 years; some reported a male predominance and others found no sex predilection. ${ }^{5}$ Our average patient age was 45 years, and we found a slight male predominance (69\%). Our most common initial imaging findings were SAH, septic infarct, and IPH. We found septic infarct to be more common than IPH and focal neurologic deficit to be a more common initial symptom than fever. Prior studies reported SAH and IPH to be the most common imaging findings and headache followed by fever as the most common presenting symptoms. ${ }^{5}$ Current literature reports the MCA as the most common location for CMAs in 50\%-70\% of patients. ${ }^{3,5}$ Similarly, we found that $78 \%$ of the CMAs were located in the MCA. We found CMAs to be multiple in $38 \%$ of patients, and this is close to prior reports of approximately $25 \%$. $^{2,3}$

Our findings are consistent with prior literature that states that both the lethality and size of CMAs during treatment are highly variable and nearly impossible to predict. A study of 18 patients with CMAs on antibiotics with a 6-week angiographic follow-up reported complete resolution in approximately $30 \%$; both enlargement and decreasing size were seen in approximately $20 \%$, and about $30 \%$ remained stable. $^{2}$ A second study reported CMAs $(10 / 20,50 \%)$ that either resolved or disappeared and 10/20 (50\%) demonstrated no change or enlargement while the patient was on antibiotic therapy. ${ }^{17} \mathrm{~A}$ third study found that approximately $30 \%$ of CMAs resolved on antibiotics alone, about 20\% decreased, $15 \%$ were unchanged, and about $20 \%$ increased in size. ${ }^{5}$ Our higher rate of clearance $(64 \%, n=7)$ for patients on antibiotics than the reported $30 \%$ average may be due to longer angiographic followup; 4 of 11 CMAs had follow-up of $>1$ year (Fig 1).

\section{When and How to Treat}

Some recommend angiography in all patients with IE who present with a focal neurologic deficit to assess the possibility of CMA; and if one is found, they recommend initial invasive treatment. Others may invasively treat only ruptured CMAs. It remains indeterminate and is currently institution- and clinician-dependent as to which approach should be used. Most CMAs (60\%) in our study were ruptured. The rupture rate of CMAs has been debated with some reporting rates from $2 \%$ to $10 \% .{ }^{18-20}$ Others report a rate of nearly $80 \% .{ }^{11,12,21}$ The sources reporting the higher rate conclude that most clinically presenting CMAs are ruptured. Septic infarct is a more likely cause of neurologic deficit in a patient with IE than CMA. Several authors think that compared with noninfectious cerebral aneurysms, CMA rupture rates are both 

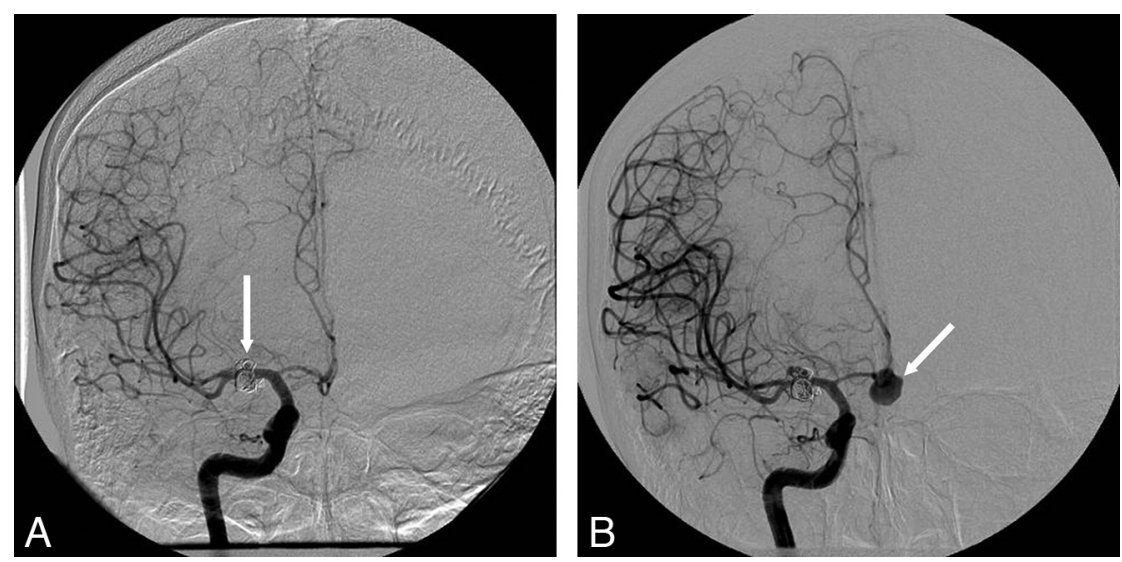

or neurosurgical repair, clipping had to be aborted in favor of coiling in 1 patient due to craniotomy complications from increased ICP. Endovascular treatment of CMAs has its own difficulties secondary to vessel wall friability, which can lead to intraprocedural wall rupture, the often irregular shape and wide neck, and their often peripheral location. Coiling is the most popular endovascular option, and this was the most common intervention in our study $(n=7)$, followed by clipping $(n=5)$. For distal MCAs, injectable polymers may be more likely to cause infarction than coils when a large arterial territory is treated, though both glue and Onyx (ev3, Irvine, California) have been successfully used. Pretreatment Wada testing may be M1 MCA aneurysm that was successfully coiled (arrow). B, A 13-mm anterior communicating artery aneurysm (arrow) that developed 7 days later. It was not treated invasively and ruptured, causing death.

higher and do not increase with size; some think that often the smaller aneurysms may be more likely to rupture. In addition, even if the aneurysm is decreasing in size while the patient is on antibiotics, rupture rates do not decrease. ${ }^{1,5,12,13}$ There have been no formal studies correlating initial CMA size with persistence. Although the number of aneurysms treated with antibiotics alone that had angiographic follow-up in our study was small $(n=11)$, initial size appeared unrelated to persistence; the largest of these CMAs completely resolved (Fig 1). If we considered mortalitybased outcomes for all 26 patients, 10 received antibiotics alone and $4 / 10(40 \%)$ died. Of the 16 who received some type of intervention, 2/16 (13\%) died; one of these deaths was due to complications from a secondary disease state.

We agree with prior recommendations that a cerebral angiogram is warranted in patients with IE who demonstrate a focal neurologic deficit or mental status change. If a CMA is found, serial angiograms should be performed to assess stability. ${ }^{4}$ Because 5 of our patients underwent acute cardiac valve-replacement surgery, 2 requiring anticoagulation reversal which delayed craniotomy, we find endovascular repair to be the treatment of choice for patients requiring acute valve replacement.

Although no complications were encountered with endovascular necessary. ${ }^{14,22}$

Neurosurgical options include clipping, ICA-ECA bypass, excision with vessel grafting, and coagulation. Cerebral mycotic aneurysms from meningitis have been successfully excised surgically but are often difficult to access postcraniotomy due to their often more central location. However, there are 2 reported successful cases of ICA-ECA bypass grafting for proximal CMAs. ${ }^{23}$ Two patients in our study were successfully treated by this method.

\section{Immunocompromised Patients}

The 3 strongly immunocompromised patients in our study demonstrated larger more rapidly growing aneurysms. The average aneurysm size was $11 \mathrm{~mm}(n=4)$ for these patients, compared with $5 \mathrm{~mm}$ $(n=34)$ in the other patients. Three of the aneurysms in strongly immunocompromised patients were successfully coiled in the acute period without complications. The 1 aneurysm in patient 7 that was not invasively treated ruptured, leading to his death. The development in patient 7 of a 13-mm anterior communicating artery aneurysm in 7 days is very similar to a case by Minnerup et al, ${ }^{8}$ who reported development of a large basilar artery CMA within 4 days in an immunocompromised patient with bacterial meningitis. Patient 24 , with invasive aspergillosis, developed a large $13-\mathrm{mm}$ cavernous
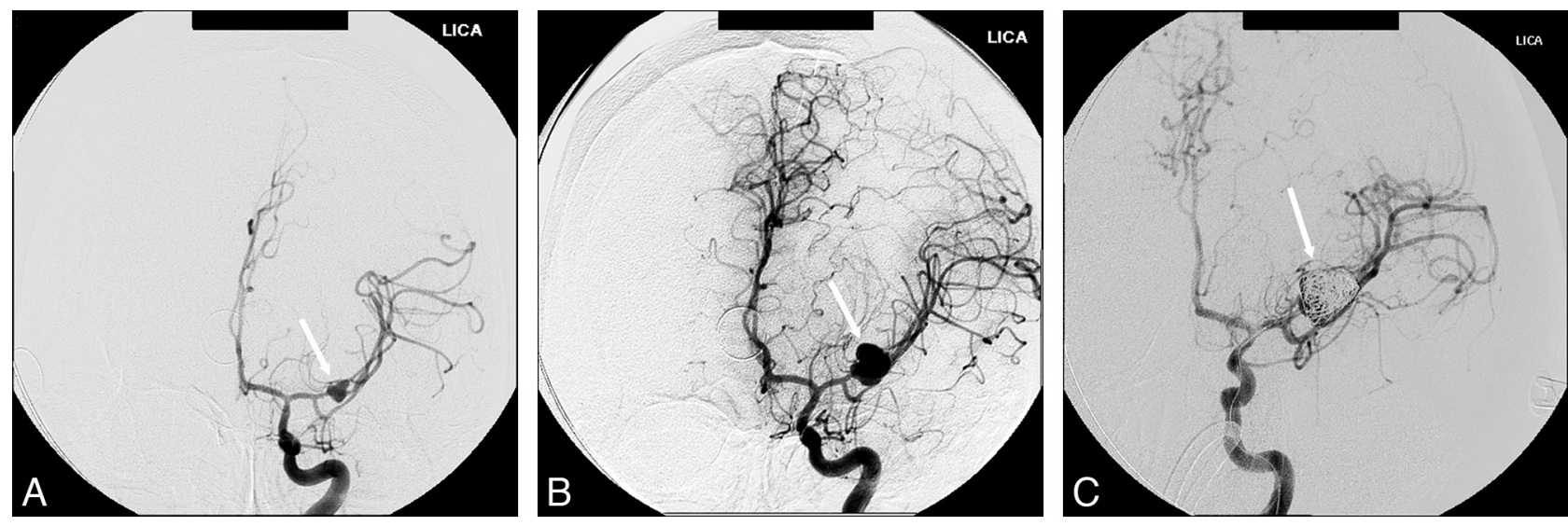

FIG 3. Catheter angiograms from patient 22 with Crohns disease on steroid immunosuppression therapy. A, Left M1 MCA multilobed 6-mm aneurysm (arrow). B, Image obtained 13 days later demonstrates growth of this aneurysm to $14 \mathrm{~mm}$ (arrow). C, Image obtained immediately postcoiling shows successful exclusion of the aneurysm (arrow). 
ICA aneurysm. Similar findings are reported in the case of an immunocompromised patient who developed a large cavernous ICA aneurysm secondary to invasive aspergillosis, which was treated successfully with coiling and balloon embolization but then expanded distally to involve the supraclinoid ICA and ruptured. ${ }^{6}$

\section{CONCLUSIONS}

In this study, we recognized a subset of patients with CMAs who are strongly immunocompromised and whose prognosis is dependent on early recognition of their immune status and early "invasive" treatment. We think that testing for immunocompromise or atypical infections should be considered in a patient with large or rapidly growing CMAs. Both endovascular and neurosurgical techniques for treatment of CMAs are safe and effective. We recommend more frequent angiographic follow-up and early invasive repair, either endovascular or neurosurgical, as the new standard of care for strongly immunocompromised patients presenting with CMAs. Three conditions that we have identified as leading to strongly immunocompromised states are AIDS, chemotherapy, and steroid immunosuppression. Endovascular repair is preferred over neurosurgical repair in patients requiring acute heart valve replacement from IE due to delays and complications with anticoagulation and prior reports of increased risk of perioperative heart failure. We found lower overall mortality for patients who received interventions (13\% mortality) versus those who received antibiotics alone (40\% mortality). We found that $64 \%$ of CMAs treated with antibiotics alone completely regressed; there was no apparent relationship between initial aneurysm size and length of persistence on antibiotics, though our sample size was small and the length of angiographic follow-up was variable.

Other limitations of this study include its retrospective design, the sequential rather than random selection of patients, and the lack of standardized angiographic follow-up. Future studies may include evaluation of the average duration of CMA persistence on antibiotics with standardized angiographic follow-up and a prospective randomized trial comparing endovascular and neurosurgical repair. Another limitation was the lack of adequate available patient history and records for a truly definitive patient classification system into "strongly" or "relatively" immunocompromised states. Future studies may examine other CMA patient risk factors leading to strongly immunocompromised states so that these patients may be stratified early for initial treatment purposes. Given the rarity of CMAs, our findings may further assist in treatment planning for patients with known or suspected CMAs, particularly in immunocompromised patients and in the acute period.

Disclosures: Laura M. Allen—RELATED: Support for Travel to Meetings for the Study or Other Purposes: Preliminary data were presented at the 2010 annual Western Neuroradiological Society meeting, and I received travel compensation (flight and hotel). Colin P. Derdeyn - UNRELATED: Consultancy: W.L. Gore and Associates, Comments: medical device company, Expert Testimony: cases related to the endovascular management of ischemic stroke and aneurysmal subarachnoid hemorrhage, Stock/Stock Options: nFocus, Pulse Therapeutics, Comments: start-ups for aneurysm and acute stroke treatment, respectively. Christopher J. Moran—RELATED: Consulting Fee or Honorarium: ev3, Codman, Boston Scientific, Comments: Consulting, speaking, and proctoring for ev3 are substantial; all are disclosed on Washington University Web site, UNRELATED: Consultancy: ev3, Codman, Boston Scientific, Comments: Consulting, speaking, and proctoring for ev3 are substantial; all are disclosed on Washington University Web site, Expert Testimony: cases, Comments: expert witness, Payment for Lectures (including service on Speakers Bureaus): ev3, Codman, Boston Scientific, Comments: Consulting, speaking, and proctoring for ev3 are substantial; all are disclosed on Washington University Web site, Payment for Development of Educational Presentations: ev3, Codman, Boston Scientific, Comments: Consulting, speaking, and proctoring for ev3 are substantial; all are disclosed on Washington University Web site.

\section{REFERENCES}

1. Kannoth S, Iyer R, Thomas SV, et al. Intracranial infectious aneurysm: Presentation, management, and outcome. J Neurolog Sci 2007;256:3-9

2. Corr P, Wright M, Handler LC. Endocarditis-related cerebral aneurysms: radiologic changes with treatment. AJNR Am J Neuroradiol 1995; 16:745-48

3. Peters PJ, Harrison T, Lennox JL. A dangerous dilemma: management of infectious intracranial aneurysms complicating endocarditis. Lancet Infect Dis 2006;6:742-48

4. Lee WK, Mossop PJ, Little AF, et al. Infected (mycotic) aneurysms: spectrum of imaging appearances and management. Radiographics 2008;28:1853-68

5. Ducruet AF, Hickman ZL, Zacharia BE, el al. Intracranial infectious aneurysms: a comprehensive review. Neurosurg Rev 2010;33:37-46

6. Hurst RW, Judkins A, Bolger W, et al. Mycotic aneurysm and cerebral infarction resulting from fungal sinusitis: imaging and pathologic correlation. AJNR Am J Neuroradiol 2001;22:858-63

7. Horten BC, Abbott GF, Porro RS. Fungal aneurysms of intracranial vessels. Arch Neurol 1976;33:577-79

8. Minnerup J, Schilling M, Wersching H, et al. Development of a mycotic aneurysm within 4 days. Neurology 2008;71:1745

9. Asaia T, Usuia A, Miyachib S, et al. Endovascular treatment for intracranial mycotic aneurysms prior to cardiac surgery. Eur J Cardiothorac Surg 2002;21:948-50

10. Erdogan HB, Erentug V, Bozbuga N, et al. Endovascular treatment of intracerebral mycotic aneurysm before surgical treatment of infective endocarditis. Tex Heart Inst J 2004;31:165-67

11. Venkatesh SK, Phadke RV, Kalode RR, et al. Intracranial infective aneurysms presenting with haemorrhage: an analysis of angiographic findings, management and outcome. Clin Radiol 2000;55:946-53

12. Chun JY, Smith W, Halbach VV, et al. Current multimodality management of infectious intracranial aneurysms. Neurosurgery 2001;48:1203-14

13. Kannoth S, Thomas SV. Intracranial microbial aneurysm (infectious aneurysm): current options for diagnosis and management. Neurocrit Care 2009;11:120-29

14. Chapot R, Houdart E, Saint-Maurice J-P, et al. Endovascular repair of cerebral mycotic aneurysms. Radiology 2002;222:389-96

15. Falagas M, Nikou S, Siempos I. Infections related to coils used for embolization of arteries: review of the published evidence. J Vasc Interv Radiol 2007;18:697-701

16. Ray WZ, Diringer MN, Moran CJ, et al. Early endovascular coiling of posterior communicating artery saccular aneurysm in the setting of Staphylococcus bacteremia: case report and review of the literature. Neurosurgery 2010;66:E847

17. Brust JC, Dickinson PC, Hughes JE, et al. The diagnosis and treatment of cerebral mycotic aneurysms. Ann Neurol 1990;27:238-46

18. Salgado AV, Furlan AJ, Keys TF. Mycotic aneurysm, subarachnoid hemorrhage, and indications for cerebral angiography in infective endocarditis. Stroke 1987;18:1057-60

19. Morawetz RB, Karp RB. Evolution and resolution of intracranial bacterial (mycotic) aneurysms. Neurosurgery 1984;15:43-49

20. van der Meulen JH, Weststrate W, van Gijn J, et al. Is cerebral angiography indicated in infective endocarditis? Stroke 1992;23:1662-67

21. Dhomne S, Rao C, Shrivasatava M, et al. Endovascular management of ruptured cerebral mycotic aneurysms. Br J Neurosurg 2008;22:46-52

22. La Barge DV 3rd, Ng PP, Stevens EA, et al. Extended intracranial applications for ethylene vinyl alcohol copolymer (Onyx): mycotic and dissecting aneurysms. Technical note. J Neurosurg 2009;111:114-18

23. Day A. Extracranial-intracranial bypass grafting in the surgical treatment of bacterial aneurysms: report of two cases. Neurosurgery 1981;9:583-88 